

\title{
RELACIÓN DE CONVERSIÓN DE ALIMENTO EN CINCO HÍBRIDOS EXPERIMENTALES DE POLLOS CAMPEROS
}

\author{
Dottavio, A. M. ${ }^{1,3}$; Fernandez, R. ${ }^{1} ;$ Antruejo, A. e. ${ }^{2}$; \\ Martines, A. ${ }^{1} ; \quad$ Canet, Z. E. ${ }^{1,4}$ \& Di Masso, R. J. ${ }^{1,3}$
}

\section{RESUMEN}

La evaluación del crecimiento dimensional de cinco híbridos experimentales de pollo campero que difieren en su genotipo materno permitió constatar la equivalencia de las poblaciones utilizadas como madre como potenciales progenitores hembra de versiones alternativas al pollo Campero INTA tradicional. Como complemento de dicha caracterización, el objetivo de este trabajo fue estudiar la relación de conversión de alimento y los caracteres asociados en los que se basa su cálculo, en los mismos cruzamientos entre los 42 y los 70 días de edad. Campero Alfa presentó el mayor aumento medio diario de peso y el mayor consumo medio diario de alimento y Campero Épsilon el menor valor promedio de ambas variables. La relación de conversión osciló entre 2,90 (Campero Épsilon) y 3,05 (Campero Alfa) $\mathrm{kg}$ de alimento por kg de peso vivo sin diferencias estadísticamente significativas en la capacidad de los diferentes grupos para transformar el alimento ofrecido en biomasa.

Palabras clave: consumo de alimento, ganancia de peso, eficiencia, sistemas semi-intensivos.

\section{SUMMARY}

Feed conversion ratio in five experimental hybrids of free range chickens.

Growth evaluation of five experimental hybrids of free-range chickens with different maternal genotype allowed to confirm the equivalence of the populations used as female parents as potential mothers for producing versions of free range chickens alternative versions of to the traditional Campero INTA chickens. As a complement of that characterization, the aim of this work was to study feed conversion ratio and body weight gain and feed intake as associated traits, from 42 to 70 days of age, in the same hybrids and in Campero INTA as the reference genotype at 42 to 70 days

1.- Cátedra de Genética. Facultad de Ciencias Veterinarias. Universidad Nacional de Rosario. Ovidio Lagos y Ruta 33. (2170) Casilda, provincia de Santa Fe.

2.- Cátedra de Producción Avícola y Pilíferos. Facultad de Ciencias Veterinarias (UNR).

3.- Carrera del Investigador Científico de la Universidad Nacional de Rosario. CIC-UNR.

4.- EEA INTA Pergamino.

Manuscrito recibido el 15 de diciembre de 2014 y aceptado para su publicación el 13 de marzo de 2015. 
of age. Campero Alfa showed the highest average daily weight gain and the highest average daily feed consumption, while Campero Epsilon showed the lowest average value of both variables. Feed conversion ratio values ranged from 2.90 (Campero Epsilon) to 3.05 (Campero Alfa) $\mathrm{kg}$ of feed consumed per kg of body weight. No statistically significant differences were observed in the ability of the different groups to transform the feed offered into biomass.

Key words: feed intake, body weight gain, feed efficiency, semi-intensive systems

\section{INTRODUCCIÓN}

Entre los principales objetivos del mejoramiento genético de las aves destinadas a la producción de carne se incluye la disminución de los costos de producción. Dado que en este tipo de explotaciones el alimento representa el principal componente de dicho costo, adquiere relevancia la eficiencia con que el mismo es transformado en biomasa. La relación de conversión es un descriptor de importancia económica que vincula la tasa de crecimiento y el consumo de alimento (O’Sullivan et al., 1992) y la disminución de la cantidad de alimento consumido por $\mathrm{kg}$ de carne producida es uno de los objetivos prioritarios en la avicultura industrial. Las particularidades de la crianza en sistemas semi-extensivos, con menores densidades que las aplicadas en la avicultura intensiva y con genotipos que presentan menor velocidad de crecimiento que el broiler industrial, determinan una menor eficiencia de conversión global de las aves producidas (Galyomitis et al., 2003). El pollo campero (Bonino, 1997), un tipo de ave de crecimiento lento que se cría en semi-cautiverio, ha sido desarrollado como una alternativa para promover una producción no tradicional para pequeños productores y cubrir la demanda de un sector de la sociedad preocupado por la calidad de sus alimentos y el bienestar animal. La evaluación de cinco poblacio- nes sintéticas utilizadas como progenitor materno en cruzamientos destinados a la producción de pollos camperos, a partir de la caracteri-zación dinámica del crecimiento dimensional de la progenie derivada de su apareamiento con gallos de una población sintética mejorada por peso corporal y eficiencia alimenticia, permitió concluir que las mismas son equivalentes como potenciales progenitores hembra en la producción de versiones de pollos camperos alternativas a Campero INTA (Dottavio et al., 2013). Como complemento de dicha caracterización, el objetivo de este trabajo fue estudiar la eficiencia de conversión de alimento y los caracteres asociados en los que se basa su cálculo, en los mismos cinco híbridos experimentales de pollos camperos (Alfa, Beta, Omega, Delta y Épsilon) con diferente estirpe materna y en el genotipo de referencia (Campero INTA), en el período comprendido entre los 42 y los 70 días de edad.

\section{MATERIALES Y MÉTODOS}

\section{AVES}

Se utilizaron machos pertenecientes a cinco grupos genéticos -Campero Alfa, Campero Beta, Campero Omega, Campero Delta y Campero Épsilon- provenientes del cruzamiento entre gallos de la población 